Bull. Korean Math. Soc. 51 (2014), No. 1, pp. 77-82

http://dx.doi.org/10.4134/BKMS.2014.51.1.077

\title{
STABILITY OF ZEROS OF POWER SERIES EQUATIONS
}

\author{
Zhinua Wang, Xiuming Dong, Themistocles M. Rassias, and Soon-Mo Jung
}

\begin{abstract}
We prove that if $\left|a_{1}\right|$ is large and $\left|a_{0}\right|$ is small enough, then every approximate zero of power series equation $\sum_{n=0}^{\infty} a_{n} x^{n}=0$ can be approximated by a true zero within a good error bound. Further, we obtain Hyers-Ulam stability of zeros of the polynomial equation of degree $n, a_{n} z^{n}+a_{n-1} z^{n-1}+\cdots+a_{1} z+a_{0}=0$ for a given integer $n>1$.
\end{abstract}

\section{Introduction}

The concept of stability of a functional equation arises when one replaces a functional equation by an inequality which acts as a perturbation of the equation. Such a problem was formulated by Ulam [16] in 1940 and solved in the next year for the case of approximately additive functions by Hyers [6]. In this case, it gave rise to the Hyers-Ulam stability for functional equations. Later, Hyers' result was generalized by T. Aoki [1] for additive mappings and by Th. M. Rassias [12] for linear mappings by allowing the Cauchy difference to be unbounded.

Since then, the stability of several functional equations has been extensively investigated by several mathematicians (see $[4,5,7,8,10,13,14]$ and references therein). The terminology Hyers-Ulam stability can also be applied to the case of other mathematical objects.

Recently, Li and Hua [11] discussed and proved the Hyers-Ulam stability of the zeros of the polynomial equation given in the form, $z^{n}+\alpha z+\beta=0$. Moreover, they raised an open problem whether the Hyers-Ulam stability also holds true for zeros of polynomial equations given in the general form (see also [2])

$$
a_{n} z^{n}+a_{n-1} z^{n-1}+\cdots+a_{1} z+a_{0}=0 .
$$

Received September 13, 2012.

2010 Mathematics Subject Classification. 39B82, 39B72.

Key words and phrases. Hyers-Ulam stability, power series equation, polynomial equation, zero.

(C)2014 The Korean Mathematical Society 
The aim of this work is to investigate the generalized of and the Hyers-Ulam stability of zeros of the power series equation (see also [3])

$$
\sum_{n=0}^{\infty} a_{n} x^{n}=0
$$

by using the fixed point theory and an idea from $[9,11]$. More precisely, we will prove that if $\left|a_{1}\right|$ is large and $\left|a_{0}\right|$ is small enough, then the zeros of the power series equation (1.2) are stable in the sense of Hyers and Ulam. As a corollary, we obtain the Hyers-Ulam stability of zeros of the polynomial equation (1.1).

\section{Generalized Hyers-Ulam stability}

In this section, we will investigate the generalized Hyers-Ulam stability of zeros of the power series equation (1.2).

Theorem 2.1. For given $\mathbb{K} \in\{\mathbb{R}, \mathbb{C}\}$, assume that $B_{r}$ is a closed ball in $\mathbb{K}$ centered at 0 and of radius $r>1 / 2$, and $p$ is a real number. Let the constants $a_{0}, a_{1}, \ldots, a_{n}, \ldots \in \mathbb{K}$ satisfy $\left|a_{1}\right|^{p} \geq\left|a_{n}\right|^{p}$ for all $n \in\{0,1, \ldots\}$,

$$
\left|a_{1}\right|>\sum_{n=2}^{\infty} n r^{n-1}\left|a_{n}\right| \text { and }\left|a_{0}\right|<\sum_{n=2}^{\infty}(n-1) r^{n}\left|a_{n}\right| .
$$

If a $y \in B_{r}$ satisfies the inequality

$$
\left|\sum_{n=0}^{\infty} a_{n} y^{n}\right| \leq \varepsilon\left(\sum_{n=0}^{\infty} \frac{\left|a_{n}\right|^{p}}{(2 r)^{n}}\right)
$$

for some $\varepsilon>0$, then there exists a zero $x \in B_{r}$ of the power series equation (1.2) such that

$$
|y-x| \leq \frac{2 r \varepsilon}{\left|a_{1}\right|^{1-p}(2 r-1)(1-\lambda)},
$$

where $\lambda=\left(1 /\left|a_{1}\right|\right) \sum_{n=2}^{\infty} n r^{n-1}\left|a_{n}\right|$ is a positive constant less than 1 and it is independent of $\varepsilon, x$ and $y$.

Proof. First, we define a function $\varphi: B_{r} \rightarrow B_{r}$ by

$$
\varphi(w)=-\frac{1}{a_{1}}\left(\sum_{n=0, n \neq 1}^{\infty} a_{n} w^{n}\right)
$$

for all $w \in B_{r}$. It follows from (2.1) that

$$
\begin{aligned}
\varphi(w) & \leq \frac{1}{\left|a_{1}\right|} \sum_{n=2}^{\infty} r^{n}\left|a_{n}\right|+\frac{\left|a_{0}\right|}{\left|a_{1}\right|} \\
& \leq \frac{1}{\left|a_{1}\right|} \sum_{n=2}^{\infty} r^{n}\left|a_{n}\right|+\frac{1}{\left|a_{1}\right|} \sum_{n=2}^{\infty}(n-1) r^{n}\left|a_{n}\right|
\end{aligned}
$$




$$
\begin{aligned}
& =\frac{1}{\left|a_{1}\right|} \sum_{n=2}^{\infty} n r^{n}\left|a_{n}\right| \\
& <r
\end{aligned}
$$

for all $w \in B_{r}$, i.e., the range of $\varphi$ is included in $B_{r}$.

We now consider the Banach space $(\mathbb{K},|\cdot|)$. Then, $B_{r}$ is a closed subset of the Banach space $\mathbb{K}$, and $\varphi$ maps $B_{r}$ into $B_{r}$. We assert that $\varphi$ is a contraction from $B_{r}$ into $B_{r}$. Indeed, it holds true that

$$
\begin{aligned}
\left|\varphi\left(w_{1}\right)-\varphi\left(w_{2}\right)\right| & =\left|\frac{1}{a_{1}}\left(-a_{0}-a_{2} w_{1}^{2}-\cdots\right)-\frac{1}{a_{1}}\left(-a_{0}-a_{2} w_{2}^{2}-\cdots\right)\right| \\
& \leq \frac{1}{\left|a_{1}\right|} \sum_{n=2}^{\infty}\left|n r^{n-1} a_{n}\right|\left|w_{1}-w_{2}\right| \\
& \leq \frac{1}{\left|a_{1}\right|} \sum_{n=2}^{\infty} n r^{n-1}\left|a_{n}\right|\left|w_{1}-w_{2}\right| \\
& =\lambda\left|w_{1}-w_{2}\right|
\end{aligned}
$$

for all $w_{1}, w_{2} \in B_{r}$, where $\lambda=\left(1 /\left|a_{1}\right|\right) \sum_{n=2}^{\infty} n r^{n-1}\left|a_{n}\right|$. In view of $(2.1), \lambda$ is a positive constant less than 1 .

According to the Banach fixed point theorem (see [15, Theorem 19.39]), there exists a unique fixed point $x$ of $\varphi$, i.e., $\varphi(x)=x$, or equivalently, $x$ satisfies (1.2). Moreover, it follows from (2.4) that

$$
\begin{aligned}
|y-x| & =|y-\varphi(y)+\varphi(y)-x| \leq|y-\varphi(y)|+|\varphi(y)-x| \\
& =|y-\varphi(y)|+|\varphi(y)-\varphi(x)| \\
& \leq\left|y-\left(-\frac{1}{a_{1}} \sum_{n=0, n \neq 1}^{\infty} a_{n} y^{n}\right)\right|+\lambda|y-x| \\
& =\frac{1}{\left|a_{1}\right|}\left|\sum_{n=0}^{\infty} a_{n} y^{n}\right|+\lambda|y-x| .
\end{aligned}
$$

Thus, by (2.2), we have

$$
\begin{aligned}
|y-x| & \leq \frac{1}{\left|a_{1}\right|(1-\lambda)}\left|\sum_{n=0}^{\infty} a_{n} y^{n}\right| \leq \frac{\varepsilon}{\left|a_{1}\right|(1-\lambda)}\left(\sum_{n=0}^{\infty} \frac{\left|a_{n}\right|^{p}}{(2 r)^{n}}\right) \\
& \leq \frac{\varepsilon}{\left|a_{1}\right|(1-\lambda)}\left(\sum_{n=0}^{\infty} \frac{\left|a_{1}\right|^{p}}{(2 r)^{n}}\right) \\
& \leq \frac{2 r \varepsilon}{\left|a_{1}\right|^{1-p}(2 r-1)(1-\lambda)}
\end{aligned}
$$

and so the results follows.

Theorem 2.2. For given $\mathbb{K} \in\{\mathbb{R}, \mathbb{C}\}$, assume that $B_{r}$ is a closed ball in $\mathbb{K}$ centered at 0 and of radius $r>1 / 2$, and $p$ is a real number. Let the constants 
$a_{0}, a_{1}, \ldots, a_{n}, \ldots, b_{0}, b_{1}, \ldots, b_{n}, \ldots \in \mathbb{K}$ satisfy $\left|a_{1}-b_{1}\right|^{p} \geq\left|a_{n}-b_{n}\right|^{p}$ for all $n \in\{0,1, \ldots\}$,

$$
\left|a_{1}-b_{1}\right|>\sum_{n=2}^{\infty} n r^{n-1}\left|a_{n}-b_{n}\right| \text { and }\left|a_{0}-b_{0}\right|<\sum_{n=2}^{\infty}(n-1) r^{n}\left|a_{n}-b_{n}\right| .
$$

If an $x \in B_{r}$ satisfies the inequality

$$
\left|\sum_{n=0}^{\infty}\left(a_{n}-b_{n}\right) y^{n}\right| \leq \varepsilon\left(\sum_{n=0}^{\infty} \frac{\left|a_{n}-b_{n}\right|^{p}}{(2 r)^{n}}\right)
$$

for some $\varepsilon>0$, then there exists a zero $x \in B_{r}$ such that $\sum_{n=0}^{\infty} a_{n} x^{n}=$ $\sum_{n=0}^{\infty} b_{n} x^{n}$ and

$$
|y-x| \leq \frac{2 r \varepsilon}{\left|a_{1}-b_{1}\right|^{1-p}(2 r-1)(1-\lambda)},
$$

where $\lambda=\left(1 /\left|a_{1}-b_{1}\right|\right) \sum_{n=2}^{\infty} n r^{n-1}\left|a_{n}-b_{n}\right|$ is a positive constant less than 1 and it is independent of $\varepsilon, x$ and $y$.

Proof. The proof runs similarly as the proof of Theorem 2.1.

\section{Hyers-Ulam stability}

In this section, we will investigate the Hyers-Ulam stability of zeros of the power series equation (1.2).

Theorem 3.1. For given $\mathbb{K} \in\{\mathbb{R}, \mathbb{C}\}$, assume that $B_{r}$ is a closed ball in $\mathbb{K}$ centered at 0 and of radius $r>0$. Let the constants $a_{0}, a_{1}, \ldots, a_{n}, \ldots \in \mathbb{K}$ satisfy

$$
\left|a_{1}\right|>\sum_{n=2}^{\infty} n r^{n-1}\left|a_{n}\right| \text { and }\left|a_{0}\right|<\sum_{n=2}^{\infty}(n-1) r^{n}\left|a_{n}\right| .
$$

If a $y \in B_{r}$ satisfies the inequality

$$
\left|\sum_{n=0}^{\infty} a_{n} y^{n}\right| \leq \varepsilon
$$

for some $\varepsilon>0$, then there exists a zero $x \in B_{r}$ of the power series equations (1.2) such that

$$
|y-x| \leq \frac{\varepsilon}{\left|a_{1}\right|(1-\lambda)},
$$

where $\lambda=\left(1 /\left|a_{1}\right|\right) \sum_{n=2}^{\infty} n r^{n-1}\left|a_{n}\right|$ is a positive constant less than 1 and it is independent of $\varepsilon, x$ and $y$.

Proof. The proof runs similarly as the proof of Theorem 2.1. 
Theorem 3.2. For given $\mathbb{K} \in\{\mathbb{R}, \mathbb{C}\}$, assume that $B_{r}$ is a closed ball in $\mathbb{K}$ centered at 0 and of radius $r>0$. Let the constants $a_{0}, a_{1}, \ldots, a_{n}, \ldots, b_{0}, b_{1}, \ldots, b_{n}$, $\ldots \in \mathbb{K}$ satisfy

$$
\left|a_{1}-b_{1}\right|>\sum_{n=2}^{\infty} n r^{n-1}\left|a_{n}-b_{n}\right| \text { and }\left|a_{0}-b_{0}\right|<\sum_{n=2}^{\infty}(n-1) r^{n}\left|a_{n}-b_{n}\right| .
$$

If an $x \in B_{r}$ satisfies the inequality

$$
\left|\sum_{n=0}^{\infty}\left(a_{n}-b_{n}\right) y^{n}\right| \leq \varepsilon
$$

for some $\varepsilon>0$, then there exists a zero $x \in B_{r}$ such that $\sum_{n=0}^{\infty} a_{n} x^{n}=$ $\sum_{n=0}^{\infty} b_{n} x^{n}$ and

$$
|y-x| \leq \frac{\varepsilon}{\left|a_{1}-b_{1}\right|(1-\lambda)},
$$

where $\lambda=\left(1 /\left|a_{1}-b_{1}\right|\right) \sum_{n=2}^{\infty} n r^{n-1}\left|a_{n}-b_{n}\right|$ is a positive constant less than 1 and it is independent of $\varepsilon, x$ and $y$.

Proof. The proof runs similarly as the proof of Theorem 3.1.

By a similar way as above, we can easily obtain the following corollary concerning the Hyers-Ulam stability of zeros of the polynomial of degree $n$, which is practically the same as a result of S.-M. Jung (see [9, Theorem 2.1]).

Corollary 3.1. For given $\mathbb{K} \in\{\mathbb{R}, \mathbb{C}\}$, assume that $B_{r}$ is a closed ball in $\mathbb{K}$ centered at 0 and of radius $r>0$. For a given integer $n>1$, let the constants $a_{0}, a_{1}, \ldots, a_{n} \in \mathbb{K}$ satisfy

$$
\left|a_{1}\right|>\sum_{i=2}^{n} i r^{i-1}\left|a_{i}\right| \text { and }\left|a_{0}\right|<\sum_{i=2}^{n}(i-1) r^{i}\left|a_{i}\right| .
$$

If $a z \in B_{r}$ satisfies the inequality

$$
\left|a_{n} z^{n}+a_{n-1} z^{n-1}+\cdots+a_{1} z+a_{0}\right| \leq \varepsilon
$$

for some $\varepsilon>0$, then there exists a zero $z_{0} \in B_{r}$ of the polynomial equation (1.1) such that

$$
\left|z-z_{0}\right| \leq \frac{\varepsilon}{\left|a_{1}\right|(1-\lambda)}
$$

where $\lambda=\left(1 /\left|a_{1}\right|\right) \sum_{i=2}^{n} i r^{i-1}\left|a_{i}\right|$ is a positive constant less than 1 and it is independent of $\varepsilon, z_{0}$ and $z$.

\section{References}

[1] T. Aoki, On the stability of the linear transformation in Banach spaces, J. Math. Soc. Japan 2 (1950), 64-66.

[2] M. Bidkham, H. A. Soleiman Mezerji, and M. Eshaghi Gordji, Hyers-Ulam stability of polynomial equations, Abstr. Appl. Anal. 2010 (2010), Article ID 754120, 7 pages.

[3] - Hyers-Ulam stability of power series equations, Abstr. Appl. Anal. 2011 (2011), Article ID 194948, 6 pages. 
[4] G. L. Forti, Hyers-Ulam stability of functional equations in several variables, Aequationes Math. 50 (1995), no. 1-2, 143-190.

[5] P. Găvruta, A generalization of the Hyers-Ulam-Rassias stability of approximately additive mappings, J. Math. Anal. Appl. 184 (1994), no. 3, 431-436.

[6] D. H. Hyers, On the stability of the linear functional equation, Proc. Natl. Acad. Sci. U.S.A. 27 (1941), 222-224.

[7] D. H. Hyers, G. Isac, and Th. M. Rassias, Stability of Functional Equations in Several variables, Birkhäuser, Basel, 1998.

[8] S.-M. Jung, Hyers-Ulam-Rassias Stability of Functional Equations in Nonlinear Analysis, Springer Optimization and Its Applications Vol. 48, Springer, New York, 2011.

[9] _ Hyers-Ulam stability of zeros of polynomial, Appl. Math. Lett. 24 (2011), no. $8,1322-1325$

[10] Pl. Kannappan, Functional Equations and Inequalities with Applications, Springer, New York, 2009.

[11] Y. Li and L. Hua, Hyers-Ulam stability of a polynomial equation, Banach J. Math. Anal. 3 (2009), no. 2, 86-90.

[12] Th. M. Rassias, On the stability of the linear mapping in Banach spaces, Proc. Amer. Math. Soc. 72 (1978), no. 2, 297-300.

[13] On the stability of functional equations and a problem of Ulam, Acta Appl. Math. 62 (2000), no. 1, 23-130.

[14] _ Functional Equations, Inequalities and Applications, Kluwer Academic, Dordrecht, 2003.

[15] E. Schechter, Handbook of Analysis and its Foundations, Academic Press, New York, 1997.

[16] S. M. Ulam, Problems in Modern Mathematics, Chapter VI, Science Editions, Wiley, New York, 1964.

ZHIHUA WANG

SCHOOL OF SCIENCE

Hubei University of Technology

Wuhan, Hubei 430068, P. R. China

E-mail address: matwzh2000@126.com

Xiuming Dong

School of ScIence

Hubei University of Technology

Wuhan, Hubei 430068, P. R. China

E-mail address: mathdxm2000@126.com

Themistocles M. Rassias

Department of Mathematics

National Technical University of Athens

Zografou Campus, 15780 Athens, Greece

E-mail address: trassias@math.ntua.gr

SOON-Mo Jung

Mathematics Section

College of Science and Technology

HONGIK UNIVERSITY

339-701 Jochiwon, Korea

E-mail address: smjung@hongik.ac.kr 